\title{
Plant diversity assessment of selected forest sites of Gaya district of Bihar, India
}

\author{
A. Chandra* \\ Forest Botany Division, Forest Research Institute, Dehradun (Uttarakhand), India \\ H. B. Naithani \\ Forest Botany Division, Forest Research Institute, Dehradun (Uttarakhand), India \\ P. K. Verma \\ Forest Botany Division, Forest Research Institute, Dehradun (Uttarakhand), India \\ J. Saxena \\ Forest Botany Division, Forest Research Institute, Dehradun (Uttarakhand), India \\ S. Prajapati \\ Forest Botany Division, Forest Research Institute, Dehradun (Uttarakhand), India \\ *Corresponding author. Email: anup8in@yahoo.com
}

\section{How to Cite}

Chandra, A. et al. (2021). Plant diversity assessment of selected forest sites of Gaya district of Bihar, India. Journal of Applied and Natural Science, 13(2), 424 - 432. https://doi.org/10.31018/jans.v13i2.2523

\begin{abstract}
Regular inventorization and monitoring of biodiversity is paramount for its conservation and sustainable utilization. Gaya district of the Bihar is endowed with rich biodiversity. For proper understanding of plant diversity, quantitative status of the vegetation is essentially required. The aim of the study was to assess different forest sites of the Gaya district of Bihar in India for their plant diversity. Study was carried out in these randomly selected eleven forest sites (Site I to Site XI). A total of 174 species belonging to 150 genera and58 families(48 Dicotyledons, 7 monocotyledons and 3 pteridophytes) were reported from all eleven study sites. Five largest families in the area were Poaceae (22 spp.), Fabaceae (14 spp.), Asteraceae (11 spp.), Rubiaceae (9 spp.) and Acanthaceae (7 spp). Different diversity attributes such as Important Value Index (IVI ), Species richness, Shannon Wiener index, Concentration of dominance and Evenness for tree, shrub and herb layers were estimated. For different sites highest IVI values for tree, shrubby and herbaceous layers were reported.Shannon wiener Index values for tree, shrubby and herbaceous layer of Nagobar site were estimated3.376, 1.986 and 2.350 respectively. Tree diversity was highest at Nagobar site whereas shrub and herb diversity was also reasonably better than most of the sites. Hence, it was revealed from the study that Nagobar site was most diverse site in the Gaya district. Study will be immense use for officials of forest department, researcher, students etc. for carrying out conservation and management work. Similar kind of study should be carried out in different forest sites of the country to get capture the status of the plant diversity for conservation and sustainable utilization.
\end{abstract}

Keywords: Diversity index, Evenness, Important value index, Species richness

\section{INTRODUCTION}

Biological diversity means the variety and variability of all living organisms from all sources including terrestrial, marine and other aquatic systems and the ecological complexes of which they are a part. This includes diversity within species and of ecosystems (McNeely et al., 1990). Human demands for food, fodder, fuel, medicine, timber, resins, and oil are directly met by biodiversity (Guar, 1999).Indirect services such as climate regulation, pollution control, soil and water conservation, nutrient cycling, pollination, and recreation (Singh, 2002; Kumar and Sharma, 2015, 2016) are also gov- erned by the biodiversity.

Environment of an area effects the vegetation of an ecosystem (Billings, 1952). The structure and function of the plant community can be understood by phytosociological study of the area. It explains and predicts pattern in a meaningful manner (Gautam and Joshi,2014). Therefore, for proper understanding of plant diversity of any area, phyto-sociology aspects should be thoroughly studied. Biodiversity in the wild state has its far reaching importance as species have diverse genotypes which can further be exploited. Forests are storehouse of the biodiversity, therefore, it is need of the hour to assess and conserve the forest 
biodiversity. However, owing to burgeoning population coupled with rapid industrialization, plant resources are under tremendous pressure. It has resulted in the decline of plant diversity in the forest ecosystem. Convention of Biological Diversity also asserted the need of regular inventorization and monitoring of biodiversity for sustainable utilization (Leadley et al. 2014).

Bihar state and adjoin Jharkhand have been extensively surveyed for its floral wealth by various workers in the past (Wood, 1903; Haines, 1921-1924: Mukharjee, 1947; Mooney, 1950; Paul, 1973; Biswas and Maheshshwari, 1980; Bhatacharya and Sarkar, 1998, Singh et al.2001etc.). Qualitative status alone cannot provide the dynamics of vegetation of the area; therefore, quantitative status should be worked out. Diversity indices for various forests have been reported by several workers (Whittakar, 1965; Risser and Rice, 1971; Ralhan et al. 1982; Knight, 1963; Peng et al., 2018; Dad, 2019).

Gaya is one of the thirty-eight districts of Bihar state, India. The district has a common boundary with the state of Jharkhand to the south. The district Gaya is situated between $24^{\circ} 30^{\prime}$ and $25^{\circ} 10^{\prime}$ north latitude and between $84^{\circ} 04^{\prime}$ and $85^{\circ} 05^{\prime}$ east longitude. The forest cover in the Gaya district is $590.31 \mathrm{~km}^{2}$ which is $11.86 \%$ of the total geographical area of the state. On the basis of density classes, $134.4 \mathrm{~km}^{2}$ comes under moderately dense forests and $455.91 \mathrm{~km}^{2}$ under open forest. There is no very dense forest in the district (FSI, 2019). To date, no work has been reported on different diversity indices of different sites of the Gaya district. Therefore, in the present study, efforts have been made to assess the plant diversity of different forest sites of Gaya District of Bihar, India.

\section{MATERIALS AND METHODS}

\section{Survey and vegetation data}

The study was conducted at the Gaya district of Bihar (Fig. 1). The climate of the district is characterized by mild cold winter, hot dry summer, hot and humid summer. Month of May is the hottest with mean maximum temperature at about $40.50^{\circ} \mathrm{C}$ and mean minimum temperature at $25.90^{\circ} \mathrm{C}$. The day temperature may go above $450^{\circ} \mathrm{C}$ in a day. January is the coldest month with man maximum temperature at $23.50^{\circ} \mathrm{C}$ and mean minimum temperature at about $8.90^{\circ} \mathrm{C}$. The average rainfall in the district is $91.3 \mathrm{~mm}$. The district gets about $88 \%$ of the normal rainfall during the monsoon months (June to September). Highest rainfall receives in the month of July, with average rainfall of $267.7 \mathrm{~mm}$. Eleven random forest sites of Gaya viz. Titri, Aamas Jhari, Patluka Murrai Pahar, Bharamyoni Mandir, North Alakdiha, South Alakdiha, Kurwa Dan Baba 2, Kurwa Dan Baba, Nagobar, Singh pursadar, Sundhaha were selected for the vegetation analysis. Random coordinating

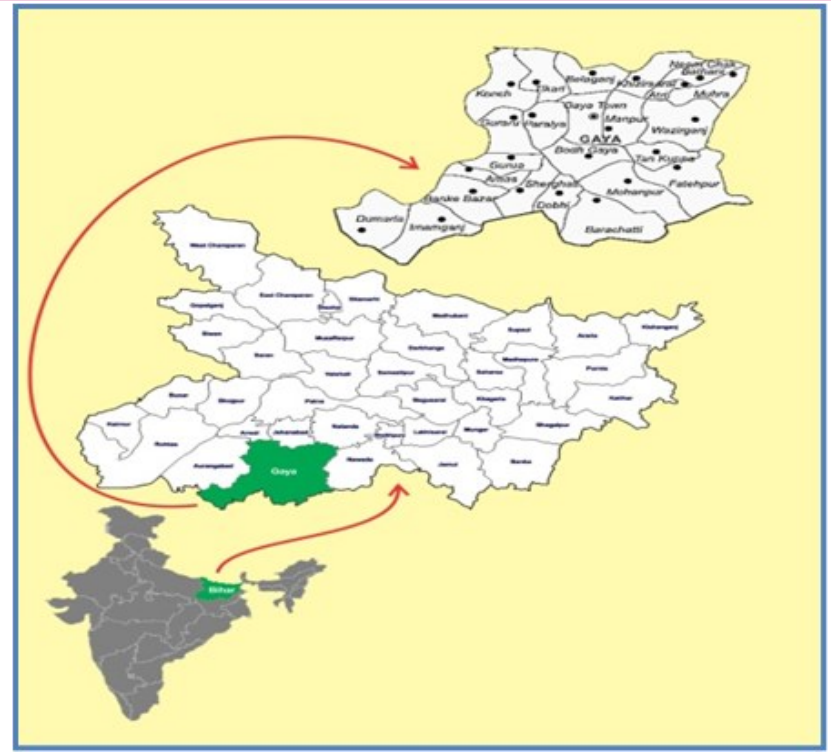

Fig. 1. Location map of study area, Gaya district.

points were provided by the GIS cell of the Forest Research Institute, Dehradun for the collection of vegetative data. Details of sites are given in Table 1.Study was carried out during 2014-15. Quantitative analysis of vegetation for frequency, density and dominance was calculated following Misra (1968). Quadrate number and size were determined; respectively by the running mean method (Kershaw, 1973) and species area curve (Misra, 1968). Ten quadrats were randomly laid in each site. Quadrate size of $10 \times 10 \mathrm{~m}, 3 \times 3 \mathrm{~m}$ and $1 \times 1 \mathrm{~m}$ was kept for trees, shrubs and herbs respectively. In each quadrat, g.b.h. (girth at breast height at $1.37 \mathrm{~m}$ above ground level) of each tree was measured and recoded individually. In the case of herb and shrub, the diameter was measured $2.5 \mathrm{~cm}$ above ground level. Species were identified with the help of concerned floras and matched with Dehradun(DD) Herbarium specimens. Plant nomenclature was updated as per the Plant List(Anonymous, 2013). Values of Relative frequency, density and dominance were summed to get Importance Value Index (IVI). Different biodiversity indices were estimated as given below:

\section{$\mathrm{S}=$ Species richness}

Total number of species

Shannon-Wiener information function (Shannon \& Wiener, 1963) was calculated using the formula:

$\mathrm{H}=-\sum \mathrm{pi} \ln \mathrm{pi}$

Where $\mathrm{pi}$ is $(\mathrm{Ni} / \mathrm{N}), \mathrm{Ni}=$ Number of individuals of species $\mathrm{i}$ and $\mathrm{N}=$ Total number of individuals of all the species.

Concentration of dominance (cd) was measured by Simpson Index (Simpson, 1949).

$\mathrm{Cd}=\Sigma(\mathrm{pi})^{2}$

Pielou's evenness index (Pielou, 1966) was calculated using formula: 
$J=H^{\prime} / \ln (S) \quad$....Eq. 3

Where $H^{\prime}$ is Shannon Weiner diversity and $S$ is the total number of species.

\section{RESULTS AND DISCUSSION}

Plant species vary in their responses to environmental factors. A given species will have a unique set of tolerances to environmental variables, such as light, temperature, moisture, and nutrients. The status of a species is an important indicator for its conservation and sustainable utilization. Importance Value Index (IVI) is a measure of how dominant a species is in a given forest area. Highest IVI species in different sites for tree, shrubby and herbaceous layers are presented in Fig. 2, 3 and 4 respectively for better understanding of plant population structure of the forest site. In tree layer, highest IVI value was estimated as 200.99 (Site-I, Butea monosperma), followed by 153.19 (Site- VII, Shorea robusta), 103.36 (Site-X, Boswellia serrata), 95.80, (Site-III, Casearia tomentosa), 93.66 (Site-IV, Sterculia urens), 69.74 (Site-VIII, Boswellia serrata), 48.09 (Site-V, Madhuca longifolia var. latifolia), 45.47 (Site-XI, Shorea robusta), 42.28 (Site-VI, Anogeissus latifolia) and 30.98 (Site-IX, Ficus benghalensis) (Fig.2). In case of shrubby layer, site I had only one dominant species i.e. Butea monosperma with IVI value of 300 . Least IVI value of 61.88 was estimated for siteVII (Pavettac rassicaulis) (Fig.3). In herbaceous layer, highest IVI value was reported of 105.91 (Site-VIII, Wrightia tinctoria) and lowest of 38.84 (Site-III, Tridex procumbens) (Fig. 4). Presence of Butea monosperma in tree as well as shrubby layer reflects the good regeneration of the plant at the site.

A total of 174 species belonging to 150 genera and 58 families (48 Dicotyledons, 7 monocotyledons and 3 pteridophytes) were reported from all eleven study sites. Five largest families in the area were Poaceae (22 spp.), Fabaceae (14 spp.), Asteraceae (11 spp.), Rubiaceae (9 spp.) and Acanthaceae (7 spp.). Habitwise, there were 71 trees (Table 2), 9 shrubs (Table 3), 20 climbers (Table 4), 49 herbs (Table 5), 20 grass, 2 sedges and 3 pteridophytes' (Table 6 ) species in all eleven sites. However, Sahu et al. (2012) recorded 57 species in dry deciduous forests of Eastern Ghats. Studies from the tropical dry deciduous forest in Sagar district reported a total of 36 trees, 8 shrubs, and 34 herbs (Thakur, 2015). A total of 29 tree species belonging to 17 families were recorded from six sites of tropical dry deciduous forests of Central India (Joshi and Dhyani, 2019) and 14 tree species under 10 families were reported from Amarkutir, tropical dry deciduous forest of West Bengal (Kumar et al., 2020). Himanshi and Jakhar (2020) reported 76 plant species belonging to 37 families from south-west Haryana. The higher number of species in the present work may be because

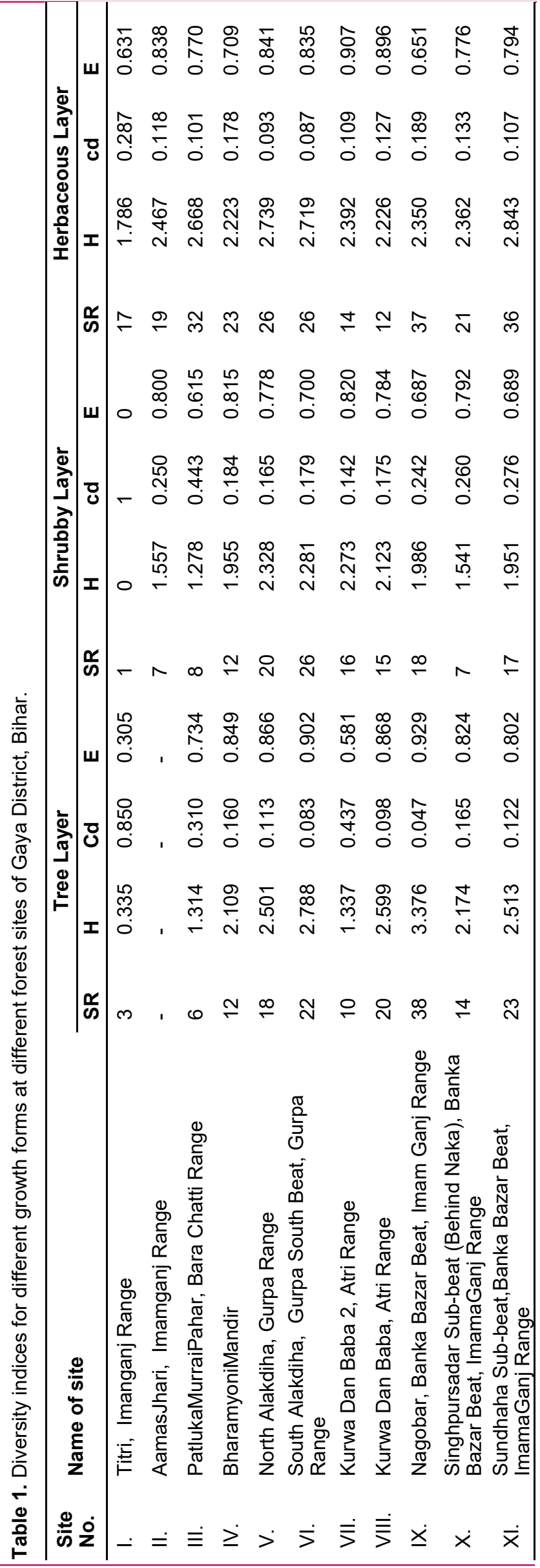


Table 2. Tree species reported from eleven forest sites of Gaya district.

\begin{tabular}{|c|c|c|c|c|c|}
\hline S.N. & Species & Family & S.N. & Species & Family \\
\hline 1. & Acacia catechu (L.f.) Willd. & Mimosaceae & 37 & Garuga pinnata Roxb. & Burseraceae \\
\hline 2 & $\begin{array}{l}\text { Adina cordifolia (Roxb.) Hook.f. } \\
\text { ex Brandis }\end{array}$ & Rubiaceae & $\begin{array}{l}38 \\
39\end{array}$ & $\begin{array}{l}\text { Gmelina arborea Roxb. } \\
\text { Grewia asiatica L. }\end{array}$ & $\begin{array}{l}\text { Verbenaceae } \\
\text { Tiliaceae }\end{array}$ \\
\hline 3 & $\begin{array}{l}\text { Aegle marmelos(L.) Corr. in } \\
\text { Trans. L. Soc. }\end{array}$ & Rutaceae & 40 & $\begin{array}{l}\text { Haplophragmaadenophyllum } \\
\text { (DC.) Dop }\end{array}$ & Bignoniaceae \\
\hline 4 & Ailanthus excelsaRoxb. & Simarubiaceae & 41 & HelicteresisoraL. & Sterculiaceae \\
\hline 5 & Alangiumchinense(Lour.) Harms & Alangiaceae & 42 & $\begin{array}{l}\text { Holarrhenapubescens(Buch.- } \\
\text { Ham.) Wall.ex.G.Don }\end{array}$ & Apocynaceae \\
\hline 6 & $\begin{array}{l}\text { Albizia lebbeck(L.) Benth.in } \\
\text { Hook }\end{array}$ & Mimosaceae & 43 & $\begin{array}{l}\text { Holoptelea integrifolia (Roxb.) } \\
\text { Planch }\end{array}$ & Ulmaceae \\
\hline 7 & $\begin{array}{l}\text { Anogeissus latifolia (Roxb. ex } \\
\text { DC.) Wall. ex Guill. \&Perr. }\end{array}$ & Combretaceae & 44 & $\begin{array}{l}\text { Hymenodictyonorixense(Roxb.) } \\
\text { Mabb. }\end{array}$ & Rubiaceae \\
\hline $\begin{array}{l}8 \\
9\end{array}$ & $\begin{array}{l}\text { Azadirachta indica A. Juss. } \\
\text { Balanites roxburghiiPlanch. }\end{array}$ & $\begin{array}{l}\text { Meliaceae } \\
\text { Balanitaceae }\end{array}$ & $\begin{array}{l}45 \\
46\end{array}$ & $\begin{array}{l}\text { Ixora pavettaAndr } \\
\text { Lagerstroemia parviflora Roxb. }\end{array}$ & $\begin{array}{l}\text { Rubiaceae } \\
\text { Lythraceae }\end{array}$ \\
\hline 10 & Bauhinia racemosaLam. & Caesalpiniaceae & 47 & $\begin{array}{l}\text { Lanneacoromandelica(Houtt.) } \\
\text { Merr. }\end{array}$ & Anacardiaceae \\
\hline $\begin{array}{l}11 \\
12\end{array}$ & $\begin{array}{l}\text { Bombax ceiba L. } \\
\text { Borassus flabellifer } \mathrm{L} .\end{array}$ & $\begin{array}{l}\text { Bombacaceae } \\
\text { Arecaceae }\end{array}$ & 48 & $\begin{array}{l}\text { Madhuca longifolia (Koenig) } \\
\text { Macbr.var.latifolia }\end{array}$ & Sapotaceae \\
\hline 13 & $\begin{array}{l}\text { Boswellia serrata Roxb. ex } \\
\text { Colebr }\end{array}$ & Burseraceae & 49 & $\begin{array}{l}\text { Mallotusphilippensis(Lam.) } \\
\text { Muell.-Arg }\end{array}$ & Euphorbiaceae \\
\hline $\begin{array}{l}14 \\
15\end{array}$ & $\begin{array}{l}\text { Bridelia retusa (L.) Spreng. } \\
\text { BuchananialanzanSpreng. }\end{array}$ & $\begin{array}{l}\text { Euphorbiaceae } \\
\text { Anacardiaceae }\end{array}$ & 50 & $\begin{array}{l}\text { Manilkara hexandra(Roxb.) } \\
\text { Dubard }\end{array}$ & Sapotaceae \\
\hline 16 & Butea monosperma(Lam.) Taub. & Fabaceae & 51 & $\begin{array}{l}\text { Miliusa tomentosa (Roxb.) } \\
\text { Finet\&Gagenpain }\end{array}$ & Annonaceae \\
\hline $\begin{array}{l}17 \\
18\end{array}$ & $\begin{array}{l}\text { Casearia graveolens Dalz. } \\
\text { Casearia tomentosa Roxb. }\end{array}$ & $\begin{array}{l}\text { Flacortiaceae } \\
\text { Flacortiaceae }\end{array}$ & 52 & $\begin{array}{l}\text { Mitragynaparvifolia(Roxb.) } \\
\text { Korth }\end{array}$ & Rubiaceae \\
\hline 19 & Cassia fistula $\mathrm{L}$. & Caesalpiniaceae & 53 & $\begin{array}{l}\text { Murrayapaniculata(L.) Jack, } \\
\text { Malayan }\end{array}$ & Rutaceae \\
\hline 20 & $\begin{array}{l}\text { Catunaregam spinosa (Thunb.) } \\
\text { Tirveng. }\end{array}$ & Rubiaceae & 54 & Nyctanthesarbor-tristis L. & Oleaceae \\
\hline 21 & $\begin{array}{l}\text { Cochlospermumreligiosum(L.) } \\
\text { Alston, Handb. Fl.Ceyl. }\end{array}$ & $\begin{array}{l}\text { Cochlosperma- } \\
\text { ceae }\end{array}$ & $\begin{array}{l}55 \\
56\end{array}$ & & $\begin{array}{l}\text { Arecaceae } \\
\text { Phyllanthaceae }\end{array}$ \\
\hline 22 & Croton roxburghiiBalak & Euphorbiaceae & 57 & Premna latifolia Roxb. & Verbenaceae \\
\hline 23 & Dalbergia sissoo Roxb. & Fabaceae & 58 & Pterocarpus marsupium Roxb. & Fabaceae \\
\hline 24 & DilleniapentagynaRoxb. & Dilleniaceae & 59 & Schleicheraoleosa(Lour.) Oken & Sapindaceae \\
\hline 25 & Diospyros cordifolia Roxb. & Ebenaceae & 60 & Semecarpus anacardium L. & Anacardiaceae \\
\hline 26 & Diospyros melanoxylonRoxb. & Ebenaceae & 61 & ShorearobustaGaertn.f., Fruct. & $\begin{array}{l}\text { Dipterocarpace- } \\
\text { ae }\end{array}$ \\
\hline 27 & $\begin{array}{l}\text { Diospyros melanoxylon } \\
\text { Roxb.var.tupru(Buch.-Ham.) } \\
\text { V.Singh }\end{array}$ & Ebenaceae & $\begin{array}{l}62 \\
63\end{array}$ & $\begin{array}{l}\text { Sterculia urensRoxb., PI. Coram } \\
\text { Sterospermumchelonoides(L.f.) } \\
\text { DC. }\end{array}$ & $\begin{array}{l}\text { Sterculiaceae } \\
\text { Bignoniaceae }\end{array}$ \\
\hline 28 & $\begin{array}{l}\text { Ehretia acuminata R.Br.var. ser- } \\
\text { rata (Roxb.) Johnston }\end{array}$ & Boraginaceae & $\begin{array}{l}64 \\
65\end{array}$ & $\begin{array}{l}\text { Streblus asper Lour. } \\
\text { Syzygiumcumini(L.) Skeels }\end{array}$ & $\begin{array}{l}\text { Moraceae } \\
\text { Myrtaceae }\end{array}$ \\
\hline 29 & EhretialaevisRoxb. & Boraginaceae & 66 & Tamarindus indica L. & Caesalpiniaceae \\
\hline 30 & Erythrina variegataL. & Fabaceae & 67 & Terminalia alataHeyne ex Roth & Combretaceae \\
\hline $\begin{array}{l}31 \\
32\end{array}$ & $\begin{array}{l}\text { Ficus arnottiana(Miq.) Miq. } \\
\text { Ficus benghalensisL. }\end{array}$ & $\begin{array}{l}\text { Moraceae } \\
\text { Moraceae }\end{array}$ & 68 & $\begin{array}{l}\text { Terminalia arjuna (Roxb. ex } \\
\text { DC.) Wight. \&Arn. }\end{array}$ & Combretaceae \\
\hline $\begin{array}{l}33 \\
34\end{array}$ & $\begin{array}{l}\text { Ficus mollisVahl } \\
\text { Ficus racemosaL. }\end{array}$ & $\begin{array}{l}\text { Moraceae } \\
\text { Moraceae }\end{array}$ & 69 & $\begin{array}{l}\text { Terminalia bellirica(Gaertn.) } \\
\text { Roxb. }\end{array}$ & Combretaceae \\
\hline 35 & Flacourtia indica (Burm.f.) Merr. & Flacortiaceae & 70 & Wrightia tinctoria (Roxb.) R.Br. & Apocynaceae \\
\hline 36 & & & 71 & ar.mauritianaLam. & Rhamnaceae \\
\hline
\end{tabular}

of more sites and large area covered under the study. Diversity indices aim to describe general properties of communities that are used to compare different regions and taxa. Diversity indices viz., Shannon-Wiener Diversity Index $(H)$, Concentration of Dominance (cd), Evenness (E) and Species Richness (SR) for different growth forms at different sites of Gaya district is presented in Table 1. The higher value of species richness indicates higher diversity of species. In the tree layer,
Nagobar site showed the highest Species Richness (SR) of 38 spp. followed by Sundhaha Sub-beat (23 spp.), South Alakdiha (22 spp.) etc. and the lowest was recorded in Titri (3 spp.). In the case of shrubby layer, the highest Species Richness (SR) value was reported for South Alakdiha (26 spp.) followed by North Alakdiha (20 spp.), Nagobar (18 spp.) etc. and lowest for Titri (1 spp.). The herbaceous layer had highest Species Richness (SR) in Nagobar (37 spp.) followed by Sundhaha 


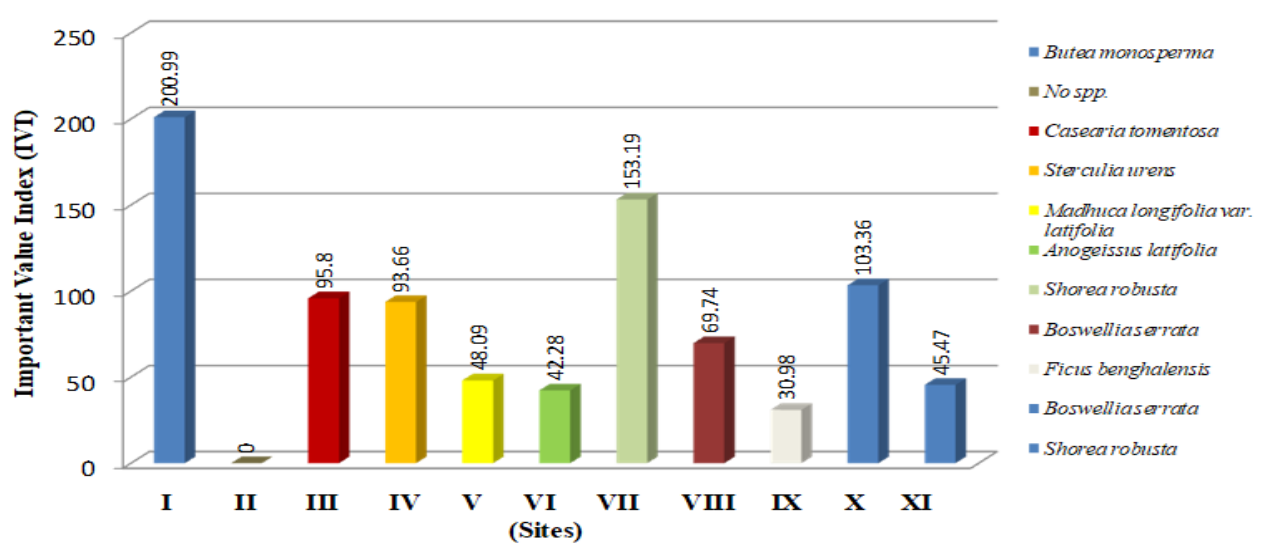

Fig. 2. Highest Important Value Index (IVI) of tree speciesin different sites (I: Titri site, II- AamasJhari, III- Patluka Murrai Pahar, IV: Bharamyoni Mandir, V: North Alakdiha, VI: South Alakdiha, VII: Kurwa Dan Baba 2, VIII: Kurwa Dan Baba, IX: Nagobar, X: Singhpursadar, XI: Sundhaha).

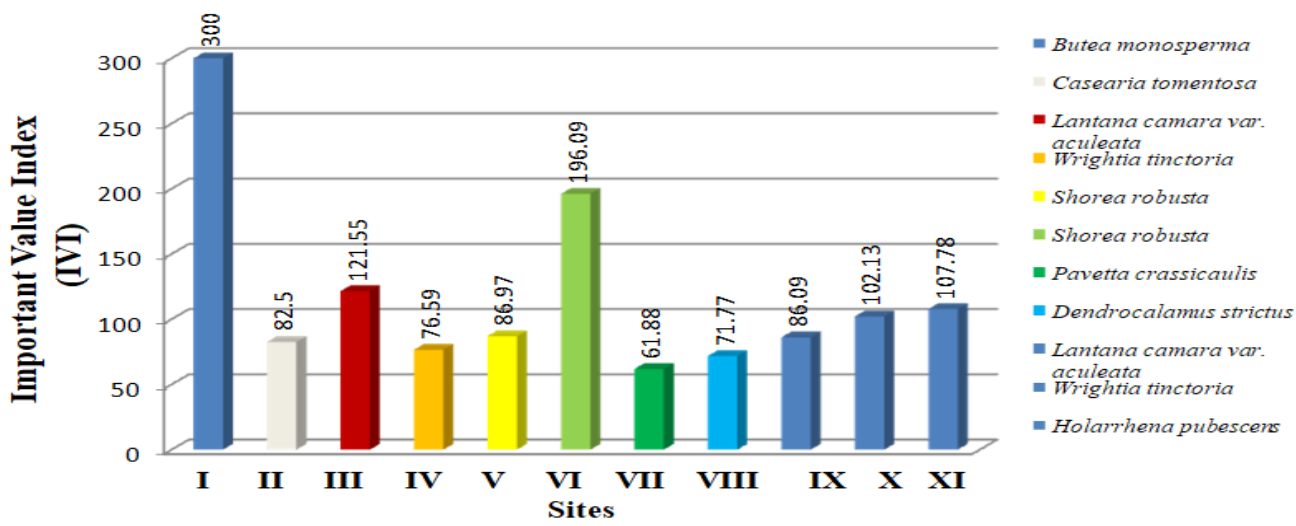

Fig. 3. Highest Important Value Index (IVI) of shrubby layer species in different sites (I: Titrisite, II- AamasJhari, III- Patluka Murrai Pahar, IV: Bharamyoni Mandir, V: North Alakdiha, VI: South Alakdiha, VII: Kurwa Dan Baba 2, VIII: Kurwa Dan Baba, IX: Nagobar, X: Singhpursadar, XI: Sundhaha).
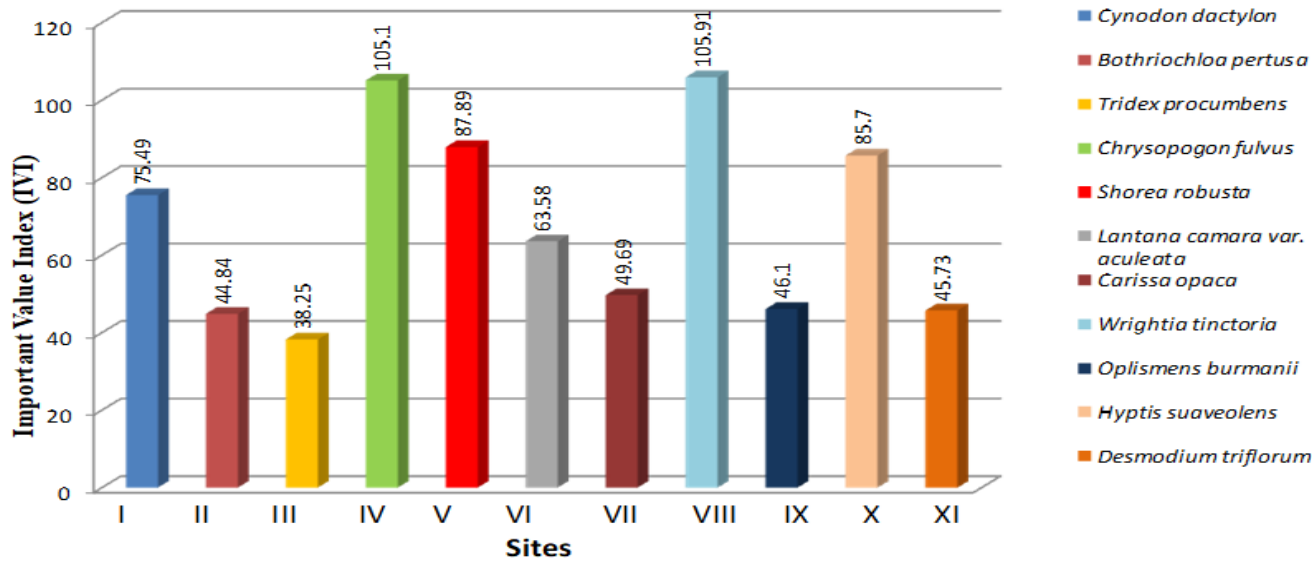

Fig. 4. Highest Important Value Index (IVI) of herbaceous layer species in different sites (I: Titri site, II- Aamas Jhari, III- Patluka Murrai Pahar, IV: Bharamyoni Mandir, V: North Alakdiha, VI: South Alakdiha, VII: Kurwa Dan Baba 2, VIII: Kurwa Dan Baba, IX: Nagobar, X: Singh pursadar, XI: Sundhaha). 
Table 3. Shrub species reported from eleven forest sites of Gaya district

\begin{tabular}{cll}
\hline S.N. & Species & Family \\
\hline 1 & $\begin{array}{l}\text { Carissa opaca Stapf ex } \\
\text { Haines }\end{array}$ & Apocynaceae \\
2 & $\begin{array}{l}\text { Clerodendrumvis- } \\
\text { cosumVent. }\end{array}$ & Verbenaceae \\
3 & $\begin{array}{l}\text { Jatropha gossypifoliaL. } \\
\text { Lantana camara L. var. }\end{array}$ & Euphorbiaceae \\
4 & $\begin{array}{l}\text { aculeata (L.) Mold. } \\
\text { Mimosa himala- } \\
\text { yanaGamble }\end{array}$ & Mimosaceae \\
6 & $\begin{array}{l}\text { Pavettacrassi- } \\
\text { caulisBremek. } \\
\text { Securinegavirosa(Roxb. } \\
\text { ex Willd.) Baill. }\end{array}$ & Rubiaceae \\
8 & $\begin{array}{l}\text { Tephrosia purpurea (L.) } \\
\text { Pers. }\end{array}$ & Fabaceae \\
9 & $\begin{array}{l}\text { Woodfordiafruticosa(L.) } \\
\text { Kurz }\end{array}$ & Lythraceae \\
\hline
\end{tabular}

Table 4. Climber species reported from eleven forest sites of Gaya district.

\begin{tabular}{|c|c|c|}
\hline S.N. & Species & Family \\
\hline 1 & AbrusprecatoriousL. & Fabaceae \\
\hline 2 & Acacia pennata (L.) Willd & Mimosaceae \\
\hline 3 & Asparagus racemosusWilld. & Liliaceae \\
\hline 4 & $\begin{array}{l}\text { Atylosiascarabaeoides(L.) } \\
\text { Benth. }\end{array}$ & Fabaceae \\
\hline 5 & Butea parviflora Roxb. & Fabaceae \\
\hline 6 & Capparis zeylanicaL. & Capparaceae \\
\hline 7 & $\begin{array}{l}\text { Cissampelos pareira L. } \\
\text { var.hirsuta(Buch.-Ham.ex } \\
\text { DC.) Forman }\end{array}$ & Menispermaceae \\
\hline 8 & Cocculus hirsutus(L.) Diels & Menispermaceae \\
\hline 9 & Combretum roxburghiiSpreng & Combretaceae \\
\hline 10 & $\begin{array}{l}\text { Dioscoreabelophylla(Prain) } \\
\text { Voigt ex Haines }\end{array}$ & Dioscoreaceae \\
\hline 11 & $\begin{array}{l}\text { Hemidesmus indicus (L.) } \\
\text { R.Br. }\end{array}$ & Asclepediaceae \\
\hline 12 & Ichnocarpus frutescens (L.) & Apocynaceae \\
\hline 13 & Ipomoea hederifoliaL. & Convolvulaceae \\
\hline 14 & $\begin{array}{l}\text { Ludwigiaoctovalvis(Jacq.) } \\
\text { Raven }\end{array}$ & Onagraceae \\
\hline 15 & $\begin{array}{l}\text { Millettia extensa (Benth.) } \\
\text { Baker }\end{array}$ & Fabaceae \\
\hline 16 & $\begin{array}{l}\text { Mucuna nigricans (Lour.) } \\
\text { Steud. }\end{array}$ & Fabaceae \\
\hline 17 & PoranapaniculataRoxb. & Convolvulaceae \\
\hline 18 & Pupalialappacea(L.) Juss & Amaranthaceae \\
\hline 19 & VentilagodenticulataWilld. & Rhamnaceae \\
\hline 20 & Ziziphus oenoplia(L.) Mill. & Rhamnaceae \\
\hline
\end{tabular}

Sub-beat (36 spp.), Patluka Murrai Pahar(32 spp.), North Alakdiha and South Alakdiha (26 spp. each) etc. and lowest in Kurwa Dan Baba (12 spp.).

In the tree layer, highest Diversity Index $(\mathrm{H})$ was observed for Nagobar (3.376) followed by South Alakdiha (2.788), Kurwa Dan Baba (2.599) etc. and lowest for
Titri (0.335). There was no tree in the AamasJhari site. In the shrubby layer, highest Diversity Index $(\mathrm{H})$ value was observed for North Alakdiha (2.328) followed by South Alakdiha (2.281), Kurwa Dan Baba- 2 (2.273) etc. and lowest for Patluka Murrai Pahar (1.278). In the herbaceous layer, the highest Diversity Index $(\mathrm{H})$ was observed for Sundhaha Sub-beat (2.843) followed by North Alakdiha (2.739), South Alakdiha (2.719) etc. and lowest for Titri (1.786). The higher value of Diversity Index $(\mathrm{H})$ indicates the variability in the type of species and heterogeneity in communities, whereas the lesser value points to homogeneity in the community. In the present study, the diversity index value range was within 0.67 to 4.03 as reported in tropical forests of the Indian subcontinent by (Kumar et al., 2010; Sundarapandian and Swamy, 2000; Verma et al., 2015, Himanshi and Jakhar, 2020).

In the tree layer, Concentration of Dominance (cd) was highest in case of Titri site $(0.850)$ followed by Kurwa Dan Baba 2(0.437), Patluka Murrai Pahar (0.310) etc. and lowest in Nagobar (0.047). The shrubby layer had highest value of Concentration of Dominance (cd) in Titri (1.000) followed by Patluka Murrai Pahar (0.443), Sundhaha Sub-beat $(0.276)$ etc. and lowest in Kurwa Dan Baba 2(0.142). In the herbaceous layer, the highest value of Concentration of Dominance (cd) was estimated for Titri (0.287) followed by Nagobar (0.187), Bharamyoni Mandir (0.178) etc. and lowest for South Alakdiha (0.087). The higher value of Concentration of Dominance (cd), the greater is the homogenous nature of community and vice-versa. In other words, such communities are dominant by single species. The lower value of the concentration of dominance indicates that the dominance of plant is shared by many species.

In the case of tree layer, highest Evenness $(E)$ value was estimated for Nagobar site (0.929) followed by South Alakdiha (0.902), Kurwa Dan Baba (0.868) etc. and lowest for Titri (0.305). In the case of shrubby layer, the highest Evenness $(E)$ value was recorded for Kurwa Dan Baba 2 site (0.820) followed by Bharamyoni Mandir (0.815), AamasJhari (0.800) etc. In the herbaceous layer, highest value of Evenness (E) was estimated for Kurwa Dan Baba 2 site (0.907) followed by Kurwa Dan Baba (0.896), North Alakdiha (0.841), Aamas Jhari (0.838) etc. and lowest for Titri (0.631). The higher value of Evenness (E) indicates that species are evenly distributed and vice-versa. In the present study, Pielou Index (E) for tree, shrubby and herbaceous layers showed a similar trend reported by different workers in tropical parts of India viz. Udaipur Rajasthan (Kumar et al. 2010), Western Ghat (Sundarapandian and Swamy, 2000), Bundelkhand region of Uttar Pradesh (Verma et al, 2015), and South West Haryana (Himanshi and Jakhar 2020). 
Table 5. Herb species reported from eleven forest sites of Gaya district.

\begin{tabular}{|c|c|c|c|c|c|}
\hline S.N. & Species & Family & S.N. & Species & Family \\
\hline 1 & Achyranthes aspera L. & Amaranthaceae & 27 & $\begin{array}{l}\text { Hyptissuaveolens (L.) } \\
\text { Poit }\end{array}$ & Lamiaceae \\
\hline 2 & $\begin{array}{l}\text { Aervasanguinolenta } \\
\text { (L.) Bl. }\end{array}$ & Amaranthaceae & & & \\
\hline 3 & Ageratum conyzoides & Asteraceae & 28 & $\begin{array}{l}\text { Indigofera linifolia(L.f.) } \\
\text { Retz. }\end{array}$ & Fabaceae \\
\hline 4 & Alysicarpus vaginalis & Fabaceae & 29 & Justicia diffusaWilld & Acanthaceae \\
\hline 5 & $\begin{array}{l}\text { (L.) DC. } \\
\text { Anisomeles indica (L.) } \\
\text { Kuntze }\end{array}$ & Lamiaceae & 30 & $\begin{array}{l}\text { Justicia simplexD. } \\
\text { Don } \\
\text { Launaea procumbens }\end{array}$ & Acanthaceae \\
\hline 6 & $\begin{array}{l}\text { Bacopa procumbens } \\
\text { (Miller) Greenman }\end{array}$ & Scrophulariaceae & 31 & $\begin{array}{l}\text { (Roxb.) Ramayya\& } \\
\text { Rajagopal }\end{array}$ & Asteraceae \\
\hline 7 & $\begin{array}{l}\text { Biophytumsensitivum } \\
\text { (L.) DC. }\end{array}$ & Oxalidaceae & 32 & $\begin{array}{l}\text { Leonotisnepetifolia(L.) } \\
\text { R.Br. }\end{array}$ & Lamiaceae \\
\hline 8 & $\begin{array}{l}\text { Blepharis repens } \\
\text { (Vahl) Roth }\end{array}$ & Acanthaceae & 33 & $\begin{array}{l}\text { Leucas plukenetii } \\
\text { (Roth) Spreng. }\end{array}$ & Lamiaceae \\
\hline 9 & $\begin{array}{l}\text { Blumeamollis(D.Don) } \\
\text { Merr. }\end{array}$ & Asteraceae & 34 & $\begin{array}{l}\text { Lindernia crustacea } \\
\text { (L.) }\end{array}$ & Scrophulariaceae \\
\hline 10 & $\begin{array}{l}\text { Blumeaobliqua(L.) } \\
\text { Druce }\end{array}$ & Asteraceae & 35 & $\begin{array}{l}\text { Nelsoniacanescens } \\
\text { (Lam.) Spreng. }\end{array}$ & Acanthaceae \\
\hline 11 & BoerhaviadiffusaL. & Nyctaginaceae & 36 & Oxalis corniculataL. & Oxalidaceae \\
\hline 12 & $\begin{array}{l}\text { Byttneriaherbacea- } \\
\text { Roxb. }\end{array}$ & Sterculiaceae & 37 & Peristrophepaniculata & Acanthaceae \\
\hline 13 & Cassia toraL. & Caesalpiniaceae & & (Forssk.) Brummit & \\
\hline 14 & $\begin{array}{l}\text { Celosia argentea L. } \\
\text { Crotalaria albida }\end{array}$ & Amaranthaceae & 38 & $\begin{array}{l}\text { Phyllanthus amarusS- } \\
\text { chum. \&Thonn. }\end{array}$ & Phyllanthaceae \\
\hline 15 & Crotalaria albıda & Fabaceae & 39 & $\begin{array}{l}\text { Phyllanthus vir- } \\
\text { gatusForst.f. }\end{array}$ & Phyllanthaceae \\
\hline 16 & $\begin{array}{l}\text { Curculigoor- } \\
\text { chioidesGaertn. } \\
\text { Cyathocline purpurea }\end{array}$ & Hypoxidaceae & 40 & $\begin{array}{l}\text { Rungiapectinata(L.) } \\
\text { Nees }\end{array}$ & Acanthaceae \\
\hline 17 & $\begin{array}{l}\text { (Buch.-Ham. ex } \\
\text { D.Don) Kuntze }\end{array}$ & Asteraceae & 41 & Sida acuta Burm & Malvaceae \\
\hline 18 & $\begin{array}{l}\text { Desmodiumtriflorum } \\
\text { (L.) DC. }\end{array}$ & Fabaceae & 42 & $\begin{array}{l}\text { Sida cordata (Burm.f.) } \\
\text { Borassum }\end{array}$ & Malvaceae \\
\hline 19 & $\begin{array}{l}\text { Dipteracanthusprostra- } \\
\text { tus(Poir.) Nees }\end{array}$ & Acanthaceae & 43 & $\begin{array}{l}\text { Borassum } \\
\text { Spermacocearticu- } \\
\text { larisL. }\end{array}$ & Rubiaceae \\
\hline 20 & ElephantopusscaberL. & Asteraceae & 44 & $\begin{array}{l}\text { Spermacocepusilla- } \\
\text { Wall. }\end{array}$ & Rubiaceae \\
\hline 21 & $\begin{array}{l}\text { Emilia sonchifolia(L.) } \\
\text { DC. }\end{array}$ & Asteraceae & 45 & Tridex procumbens L. & Asteraceae \\
\hline 22 & $\begin{array}{l}\text { Eriocaulon quinquan- } \\
\text { gulareL. }\end{array}$ & Eriocaulaceae & 46 & $\begin{array}{l}\text { Triumfettarhomboi- } \\
\text { deaJacq. }\end{array}$ & Tiliaceae \\
\hline 23 & Euphorbia hirtaL. & Euphorbiaceae & & $\begin{array}{l}\text { Urena sinuate L. } \\
\text { subsp.sinuata(L.) }\end{array}$ & \\
\hline 24 & $\begin{array}{l}\text { Evolvulus- } \\
\text { alsinoides (L.) L }\end{array}$ & Convolvulaceae & 47 & Borassum var.sinuata. & Malvaceae \\
\hline 25 & $\begin{array}{l}\text { Evolvulusnummularius } \\
\text { (L.) L }\end{array}$ & Convolvulaceae & 48 & Vernonia cinerea (L.) & Asteraceae \\
\hline 26 & $\begin{array}{l}\text { Gnaphalium pensyl- } \\
\text { vanicum Willd. }\end{array}$ & Asteraceae & 49 & Vicoa indica (L.) DC. & Asteraceae \\
\hline
\end{tabular}

\section{Conclusion}

It reveals from the study that forest sites of Gaya district possess a significantly high floristic diversity. On the basis of different diversity attributes viz. species richness, diversity index, the concentration of dominance and evenness in the tree layer, Nagobar site is the most diverse site followed by South Alakdiha, Kurwa Dan Baba, North Alakdiha etc. where AamasJhari site is devoid of any tree species. Shrub and herb di- versity was also considerably higher than most of the sites in Nagobar site. The low diversity of tree species indicates the disturbance in sites such as AamasJhari, Titri, and Patluka Murrai Pahar. Anthropogenic activities such as tree felling for fuelwood and timber, grazing, encroachment, etc., may be factors for the depletion of plant diversity. Hence, suitable management strategiesshould be developed for the improvement of the diversity of the tree species in the low diversity sites. Villagers living in the fringes of forests should be 
Table 6. Sedges, Grass and Pteridophytes reported from eleven forest sites of Gaya district.

\begin{tabular}{|c|c|c|}
\hline S.N & Species & Family \\
\hline \multicolumn{3}{|c|}{ Sedges } \\
\hline 1 & Cyperus niveusRetz. & Cyperaceae \\
\hline 2 & Cyperus rotundusL. & Cyperaceae \\
\hline \multicolumn{3}{|c|}{ Grasses } \\
\hline 1 & ApludamuticaL. & Poaceae \\
\hline 2 & Aristida adscensionisL. & Poaceae \\
\hline 3 & Bothriochloapertusa(L.) & Poaceae \\
\hline 4 & \multirow{2}{*}{$\begin{array}{l}\text { Brachiariaramosa(L.) Stapf } \\
\text { Brachiariareptans(L.) } \\
\text { C.A.Gardner\&C.E.Hubb. }\end{array}$} & Poaceae \\
\hline 5 & & Poaceae \\
\hline \multirow[t]{2}{*}{6} & \multirow{2}{*}{$\begin{array}{l}\text { Chrysopogon fulvus (Spr.) } \\
\text { Chiov. } \\
\text { Cymbopogon khasianus }\end{array}$} & Poaceae \\
\hline & & \\
\hline 7 & $\begin{array}{l}\text { (Munro ex Hack.) Stapf ex } \\
\text { Bor }\end{array}$ & Poaceae \\
\hline 8 & \multirow{2}{*}{$\begin{array}{l}\text { Cynodondactylon(L.) Pers. } \\
\text { Dactylocteniumaegyptium(L.) } \\
\text { Willd. }\end{array}$} & Poaceae \\
\hline 9 & & Poaceae \\
\hline 10 & $\begin{array}{l}\text { Dendrocalamusstrictus } \\
\text { (Roxb.) Nees }\end{array}$ & Poaceae \\
\hline 11 & \multirow{3}{*}{$\begin{array}{l}\text { Desmodiumgangeticum(L.) } \\
\text { DC. } \\
\text { Desmodiumtriflorum(L.) DC. } \\
\text { Dichanthiumannulatum } \\
\text { (Forssk.) Stapf }\end{array}$} & Poaceae \\
\hline 12 & & Poaceae \\
\hline 13 & & Poaceae \\
\hline 14 & $\begin{array}{l}\text { Eragrostiellanardoides(Trin.) } \\
\text { Bor }\end{array}$ & Poaceae \\
\hline 15 & $\begin{array}{l}\text { Eragrostistenella(L.) P.Beauv. } \\
\text { ex Roem. \&Schult. }\end{array}$ & Poaceae \\
\hline 16 & \multirow{2}{*}{$\begin{array}{l}\text { Heteropogoncontortus(L.) } \\
\text { Melanocenchrisjacquemontii- } \\
\text { Jaub. \&Spach. }\end{array}$} & Poaceae \\
\hline 17 & & Poaceae \\
\hline 18 & $\begin{array}{l}\text { Oplismenusburmannii(Retz.) } \\
\text { P. Beauv }\end{array}$ & Poaceae \\
\hline 19 & $\begin{array}{l}\text { Pennisetum glaucum (L.) R. } \\
\text { Br. } \\
\text { Sporobolus indicus (L.) R. Br. }\end{array}$ & Poaceae \\
\hline 20 & $\begin{array}{l}\text { var.diander(Retz.) Jovet. \& } \\
\text { Guedes }\end{array}$ & Poaceae \\
\hline \multicolumn{3}{|c|}{ Pteridophytes } \\
\hline 1 & \multirow{3}{*}{$\begin{array}{l}\text { Adiantum incisumForssk. } \\
\text { Cheilanthesfarinosa(Forssk.) } \\
\text { Kaulf. } \\
\text { Lygodiumflexuosum (L.) Sw. }\end{array}$} & Adiantaceae \\
\hline 2 & & Cheilanthaceae \\
\hline 3 & & Lygodiaceae \\
\hline
\end{tabular}

made aware of the importance of forest biodiversity conservation and its sustainable utilization through a mass awareness programme. The finding of the study will be useful for researchers and officials of the State Forest Department for formulation and implementation of future management study of the area.

\section{ACKNOWLEDGEMENTS}

The authors are thankful to the Director, Forest Research Institute, Dehradun, for constant encouragement and support. The authors are also thankful to the Principal Chief Conservator of Forests (PCCF) and other Forest officials of Bihar Forest Department for funding and providing the necessary support for carrying out the study.

\section{Conflict of interest}

The authors declare that they have no conflict of interest.

\section{REFERENCES}

1. Anonymous (2013). The Plant List, Version 1.1., http:www.theplantlist.org

2. Bhatacharya, P. K. \& Sarkar K. (1998). Flora of WestChamparan District, Bihar. Botanical Survey of India, Calcutta.

3. Billings, W.D. (1952). The environment complex in relation to plant growth and distribution Quarterly Review of Biology, 27, 251-265.

4. Biswas, D.K. \& Maheshwari, J. K. (1980). A contribution to the vegetation of Chaibasa, Singhbhum District in South Bihar. Bull. Bot. Soc. Bengal, 25 (1 \& 2): 43-51.

5. Dad, J.M. (2019). Phytodiversity and medicinal plant distribution in pasturelands of North Western Himalaya in relation to environmental gragients. J. Mt. Science. 16, 884897.

6. Forest Survey of India. (2019). State of Forest Report. Forest Survey of India, Dehradun, India

7. Gaur, R.D. (1999). Flora of the District Garhwal Northwest Himalaya: with Ethanobotanical Notes. TransMedia, Srinagar, U.P., India.

8. Gautam, M. \& Joshi, S.P. (2014). Analysis of vegetation dynamics and phytodiversity from three dry deciduous forests of Doon Valley, Western Himalaya, India. Journal of Asia-Pacific Biodiversity, 7, 292-304.

9. Haines, H.H. (1921-24). The Botany of Bihar and Orissa. Adlard and Sons, London.

10. Himanshi, H. \& Jakhar, S. (2020). Floristic diversity and vegetation analysis of the communilty forests of South West Haryana, India. Current Botany, 11, 51-59, https// doi:10.25081/cb.2020.v11.6032.

11. Joshi, R.K., Dhyani, S. (2019). Biomass, carbon density and diversity of tree species in tropical dry deciduous forests in Central India. Acta EcologicaSinica., 39(4), 289299.

12. Kershaw, K.A. (1973). Quantitative and Dynamic Plant Ecology. (London: Edward Arnold Ltd.) 308pp.

13. Knight, D.H. (1963). A distance method for constructing forest profile diagrams and obtaining structural data. Tropical Ecology,4, 89-94.

14. Kumar, J.I.N., Kumar, R.N., Bhoi, R.K. \& Sajish, P.R. (2010). Tree species diversity and soil nutrient status of tropical dry deciduous forest of western India. Tropical Ecology. 51(2), 273-279.

15. Kumar, A. \& Sharma, M.P. (2015). Estimation of carbon stock of balgangaReserve forest, Uttarakhand. India. For. Sci. Technol. 11 (4), 177-181.https//doi: 10.1080/21580 103.2014.990060.

16. Kumar, A. \& Sharma, M.P. (2016). Carbon stock estimation in the catchment of Kotli Bhel $1 \mathrm{~A}$ Hydroelectric reservoir, Uttarakhand, India. Ecotoxicol. Environ. Saf., 134: 365-369.

17. Kumar, M.L., Nag, A., Malakar, S., Joshi, H.G. (2020). Population Structure and Diversity of Trees in Amarkutir, A Tropical Dry Deciduous Forest of West Bengal, India. Indian Journal of Ecology. 47(1), 150-154.

18. Leadley, P.W., Krug, C.B., Alkemade, R., Pereira, H.M., 
Sumaila U.R., Walpole, M., Marques, A., Newbold, T., Teh, L.S.L, van Kolck, J., Bellard, C., JanuchowskiHartley, S.R. \& Mumby, P.J. (2014): Progress towards the Aichi Biodiversity Targets: An Assessment of Biodiversity Trends, Policy Scenarios and Key Actions. Secretariat of the Convention on Biological Diversity, Montreal, Canada. Technical Series 78, 500 pages.

19. McNeely, G., Mille, K.R., Reid, W.V., Mittermeier, R.A. \& Werner, T.R. (1990). Conserving the World's Biological Diversity. IUCN, Gland.

20. Misra, R. (1968). Ecological Workbook. Oxford Press, New Delhi.

21. Mooney, H.F. (1950). Supplement to the Botany of Bihar and Orissa. Catholic Press, Ranchi

22. Mukherjee, S.K. (1947). A Botanical Tour in Chhotanagpur. Bull. Bot. Soc. Bengal, 1:27-28.

23. Paul, S.R. (1973). On the aquatic and Marsh Flora of Monghyr, Bihar. Botanique, 143-152.

24. Peng, Y., Fan, M., Song, J., Cui, T. \& Li, R. (2018). Assessment of plant species diversity based on hyperspectral indices at a fine scale. Scientific Reports, 8 (1).

25. Pielou, E.C. (1966). The measurement of diversity in different types of biological collections. Journal of Theoretical Biology, 13, 131-144.

26. Ralhan, P.K., Saxena, A. K. \& Singh, J. S. (1982). Analysis of forest vegetation at and around Nainital in Kumaun Himalaya. Proc. Indian National Sciences, 19, 307-324.

27. Risser, P.G. \& Rice, E.L. (1971). Diversity in tree species in Oklahoma upland forests. Ecology, 52, 876-880.

28. Sahu, S.C., Dhal, N. K. \& Mohanty, R.C. (2012). Tree species diversity, distribution and population structure in a tropical dry deciduous forest of Malygiri hill range, Eastern India. Tropical Ecology. 2012; 53(2),163-168.

29. Shannon, C. E. \& Wiener, W. (1963). The Mathematical Theory of Communities. University of Illinois press, Urbana.

30. Simpson, E. M. (1949). Measurement of diversity. Nature, $163,688$.

31. Singh, J.S. (2002). The biodiversity crisis: a multifaceted review. Curr. Sci. 82 (6), 638-647.

32. Singh, N.P., Mudgal V., Khanna, K.K, Srivastava, S.C., Sahoo, A. K., Bandhopadhay, S., Aziz, N., Das M. Bhattacharya, R.P. \& Hajra, P.K. (2001). Flora of BiharAnalysis. Botanical Survey of India, Calcutta

33. Sundarapandian, S.M. \& Swamy, P.S. (2000). Forest ecosystem structure and composition along an altitudinal gradient in the Western Ghats, South India. Journal of tropical forest Science, 12,104-123.

34. Thakur, A.S. (2015). Floristic composition, life-forms and biological spectrum of tropical dry deciduous forest in Sagar Districts, Madhya Pradesh, India. Tropical Plant Research, 2(2),112-119.

35. Verma, M.K., Niranjan, R.K. \& Pal, A. (2015). Phytosociological attributes of a tropical dry deciduous forest of Bundelkhand region of Uttar pradesh, India. Journal of Biodiversity and Environmental Sciences, 3 (10), 86-89.

36. Whittakar, R.H. (1965). Dominance and diversity inland plant communities: numerical relations of species express in importance of competition in community function and evolution. Science, 147 (3655): 250-260.

37. Wood, J. J. (1903). Plants of Chotanagpur including Jaspur and Surguza. Rec. Bot. Sur. India. 2 2(1),170. 